\title{
Intestinal carriage of Extended Spectrum Beta-Lactamase producing $E$. coli in women with urinary tract infections, Cameroon
}

\author{
Ingrid Cécile Djuikoue ${ }^{1,2}$, Paul-Louis Woerther ${ }^{3}$, Michel Toukam ${ }^{1,2 \#}$, Charles Burdet ${ }^{4,5 \#}$, Etienne Ruppé6,\#, \\ Kamga Hortense Gonsu ${ }^{2,7}$, Charles Fokunang ${ }^{2}$, Assiya EI Mniai ${ }^{6}$, Kamgue Larissa ${ }^{2}$, Anatole Constant \\ Pieme $^{2}$, Mallila Georgia Mboupaing ${ }^{2}$, Caroline Mietchop Kakam², Hervé Kengne Fogang ${ }^{2}$, Antoine \\ Andremont $^{5,6}$, Jeanne Ngogang ${ }^{2}$ \\ ${ }^{1}$ Laboratoire de Microbiologie, Université des Montagnes, Bangangté, Cameroun \\ ${ }^{2}$ Département de Microbiologie, Faculté de Médecine et de Sciences Biomédicales, Université de Yaoundé I, \\ Yaoundé, Cameroun \\ ${ }^{3}$ Laboratoire de Microbiologie, Institut Gustave-Roussy, Villejuif, France \\ ${ }^{4}$ Service des Maladies Infectieuses et Tropicales, Hôpital Bichat-Claude Bernard, AP-HP, Paris, France \\ 5 UMR 1137 INSERM, (IAME) Université Paris-Diderot, Paris, France \\ ${ }^{6}$ Laboratoire de Bactériologie, Hôpital Bichat-Claude Bernard, AP-HP, Paris, France \\ ${ }^{7}$ Laboratoire de Bactériologie, Centre Hospitalier Universitaire de Yaoundé, Yaoundé, Cameroun
}

\# These authors contributed equally to the present work.

\begin{abstract}
Introduction: During the last decade, the prevalence of the intestinal carriage of extended spectrum beta-lactamases - producing Escherichia coli (ESBL-E. coli) has continued to increase worldwide in the community, especially in developing countries. Hence, we undertook a study to determine the ESBL-E. coli fecal carriage rate and the associated risk factors in Cameroonian women.

Methodology: A total of 86 women suspected of community-acquired urinary tract infections (UTI) were included in 10 health structures from May 2011 to April 2012. After filling a questionnaire, they provided a stool sample that was plated on selective media for ESBL producing bacteria. The identification of strains was obtained with mass spectrometry and the antibiotic susceptibility by disk diffusion in agar media. The ESBL type was determined by PCR. The relative abundance of ESBL-E. coli was measured for positive samples. Eventually, the presence of antibiotics in stool was assessed.

Results: The carriage rate of ESBL-E. coli was 57/86 (66.3\%). Phenotypic and molecular characterization showed that all ESBL-E. coli strains contained group 1 CTX-M enzymes. Multivariate analysis showed that ESBL-E. coli fecal carriage was associated with the presence of antibiotics in stools $(\mathrm{p}<0.05)$. Although not significant, mean ESBL relative abundance tended to be higher in patients with antibiotic exposure. Conclusions: Our results show that the carriage of ESBL-E. coli fecal carriage in women with UTI suspicion from the Cameroonian community is extremely high and associated with recent antibiotic intake.
\end{abstract}

Key words: ESBL; carriage; UTI; Cameroon; community.

J Infect Dev Ctries 2016; 10(10):1135-1139. doi:10.3855/jidc.7616

(Received 01 September 2015 - Accepted 30 November 2015)

Copyright (C) 2016 Djuikoue et al. This is an open-access article distributed under the Creative Commons Attribution License, which permits unrestricted use, distribution, and reproduction in any medium, provided the original work is properly cited.

\section{Introduction}

The global dissemination of extended spectrum beta-lactamase (ESBL)-producing Escherichia coli (ESBL-E. coli) is a major public health concern. The ESBL diffusion is mainly due to the rapid and global spread of plasmid-borne bla $a_{\text {CTX-M }}$ genes in E. coli which is the most common and abundant Enterobacteriaceae in the human intestinal gut [1]. Nowadays, the prevalence of ESBL- $E$. coli carriage in the community setting is estimated to range between $10 \%$ and $50 \%$ according to the geographical areas. This prevalence has been especially observed to be the highest in developing countries, as a possible result of poorly controlled antibiotic consumption and suboptimal hygiene conditions [2]. Although limited to a few reports, data originating from Africa's WHO region show that the carriage of ESBL-E. coli ranged from 5 to $30 \%$ according to the country and the studied populations [2]. A recent study in Cameroon reported that the intestinal carriage of ESBL-producing Enterobacteriaceae was as high as $23.1 \%$ [3]. In this context, we designed the present study in order to assess ESBL-E. coli fecal carriage rate in female patients with 
suspicion of urinary-tract infection (UTI) in Yaoundé, Cameroon.

\section{Methodology}

Population

A cross-sectional descriptive study for which ethical clearance was obtained from the Cameroonian National Ethics Committee (approval No. 207/CNE/SE/2011) was carried out between May 2011 and April 2012 in 10 health structures in Yaoundé (Cameroon), including 3 public hospitals (General Hospital, Central Hospital and Yaoundé Gynecology, Obstetrics and Pediatric Hospital) and 7 private clinics. Outpatient women consulting for suspicion of UTI were approached by one of the investigators who provided information about the study. When the patient agreed to participate and gave her consent, she provided a freshly passed (less than 6hrs) fecal sample in the provided sterile container and answered a standardized anonymous questionnaire on clinical (including history of UTIs, antibiotic use and hospitalization during the last three months, diabetes and pregnancy) and demographic data (age, address, occupation, marital status, number of children). Stool samples were kept at $4^{\circ} \mathrm{C}$ and transported to the CREMER laboratory of the Ministry of Scientific Research Cameroon within the same day, where they were $1 / 10$ diluted in brain heart infusion broth supplemented with $10 \%$ glycerol and frozen at $-80{ }^{\circ} \mathrm{C}$. The diluted samples were later airtransported in dry ice to the bacteriology laboratory of Bichat-Claude Bernard Hospital in Paris (France) for further analysis.

\section{Microbiological analysis}

Samples were thawed in batches, plated on Drigalski agar (Oxoid, Dardilly, France) supplemented or not with cefotaxime $1 \mathrm{mg} / \mathrm{L}$ (Mylan Pharmaceuticals, Saint-Priest, France) and incubated for 48 hours at $37^{\circ} \mathrm{C}$. All distinct colonies (with respect to the colour and aspect of the colonies) that could grow on Drigalski agar with cefotaxime were subcultured on trypticase soy agar (Bio-Rad, Marne-la-Coquette, France) and identified by mass spectrometry (Bruker Daltonics, Bremen, Germany). E. coli isolates were tested for susceptibility to amoxicillin, tircacillin, amoxicillin + clavulanic acid, cefotaxime, cefoxitin, ceftazidime, cefepime, ertapenem, nalidixic acid, ciprofloxacin, gentamicin, amikacin, fosfomycin and co-trimoxazole (Bio-Rad, Marne-la-Coquette, France) using the disc diffusion method and presence of ESBL was determined using the double disc-diffusion phenotypic method, as recommended by the Antibiogram
Committee of the French Society for Microbiology [4]. To confirm the ESBL identification with molecular methods, total DNA was extracted from $E$. coli strains, as described [5] and $b l a_{\mathrm{CTX}-\mathrm{M}}$ (group 1), bla $a_{\mathrm{TEM}}$ and $b l_{\mathrm{SHV}}$ genes were amplified by PCR using specific primers, as described [6].

Exposition to antibiotics at the time of fecal sampling was defined by the detection of fecal antimicrobial activity, as described [7]. Briefly, $10 \mu \mathrm{L}$ of each defrosted stool sample was placed on antibiotic free sterile $6 \mathrm{~mm}$ diameter paper discs (Dutscher, Brumath, France). The discs were then placed on Mueller-Hinton agar (Oxoid, Dardilly, France) containing a $10^{5} \mathrm{CFU} / \mathrm{mL}$ suspension of a fully susceptible E. coli strain (strain 25922, derived from the American Type Culture Collection, Manassas, VA, USA). Stool samples for which a zone of inhibition was observed around the disc following overnight incubation at $37^{\circ} \mathrm{C}$ were considered positive and the corresponding patient as exposed to antibiotics at the time of sampling.

Densities of total Enterobacteriaceae and of ESBLE. coli were determined by plating serial dilutions of the broth on Drigalski agar, with or without $1 \mathrm{mg} / \mathrm{L}$ cefotaxime. CFUs were counted in decimal logarithms at the dilution in which 1-100 CFUs grew. ESBLrelative abundance (ESBL-RA) was calculated as the ratio of the ESBL-E. coli counts divided by the total number of Enterobacteriaceae [8]. For women who carried more than one ESBL E. coli, only the ESBL-RA of the dominant clone was considered.

\section{Statistical analysis}

We compared demographic and clinical characteristics in patients according to fecal ESBL-E. coli carriage. Characteristics tested included: season during which the suspicion of UTI occurred (dry/rainy), city of origin (Yaoundé/other), patient's age, history of diabetes or pregnancy, use of antibiotics in the 3 months preceding the inclusion, hospitalization in the 3 months preceding the inclusion, retained diagnosis of UTI and antimicrobial activity detected in the stool. Comparisons between groups were performed using nonparametric tests (Wilcoxon or Fisher exact tests).

We searched for risk factors associated with fecal ESBL-E. coli carriage. Variables achieving $P<0.20$ in nonparametric tests were considered for a multivariate logistic regression analysis to identify risk factors of fecal ESBL-E. coli carriage. Using a forward selection method, we obtained a final model in which all risk factors had $P<0.05$. First-order interaction was tested for significant variables. The model discrimination was 
assessed by the c-statistic and its 95\% confidence interval $(95 \% \mathrm{CI})$, and the model calibration was assessed by the Hosmer-Lemeshow goodness-of-fit test. Analyses were performed with SAS v9.3 (SAS Institute Inc., Cary, NC). All tests were 2-sided with a type-I error fixed to 0.05 .

\section{Results}

A total of 86 patients provided a stool sample and were included. An E. coli isolate was identified from their stool on cefotaxime containing plates in 57 $(66.3 \%)$ of them, including 5 carrying two E. coli strains with different morphologies and antibiotic susceptibility patterns. This ended in a total of $62 \mathrm{E}$. coli strains. All the 62 E. coli exhibited an ESBL phenotype and were tested positive for the CTX-M group 1 by PCR. ESBL-E. coli rates of resistance to non-betalactam antibiotics tested were as follows: ciprofloxacin $(\mathrm{n}=56,90 \%)$, gentamicin $(\mathrm{n}=25,41 \%)$, amikacin $(\mathrm{n}=$ $8,13 \%)$ and co-trimoxazole $(\mathrm{n}=62,100 \%)$. Of note, all were susceptible to fosfomycin.

Among the 86 included patients, 43 (50\%) were exposed to antibiotics at the time of sampling, as demonstrated by the detection of an antimicrobial activity in the fecal sample.

As shown in Table 1, fecal ESBL-E. coli carriage was not different according to age, pregnancy, antibiotics use during the last 3 months and hospitalization the last 3 months. Univariate analysis (Table 1) showed that detection of an antimicrobial activity in the stool sample was significantly associated with ESBL-E. coli carriage versus non carriage $(P=0.001)$. Multivariate analysis showed that the detection of antimicrobial activity in the stool sample
Figure 1. Extended-spectrum beta-lactamase (ESBL) relative abundance with regards to the antibiotic exposure.

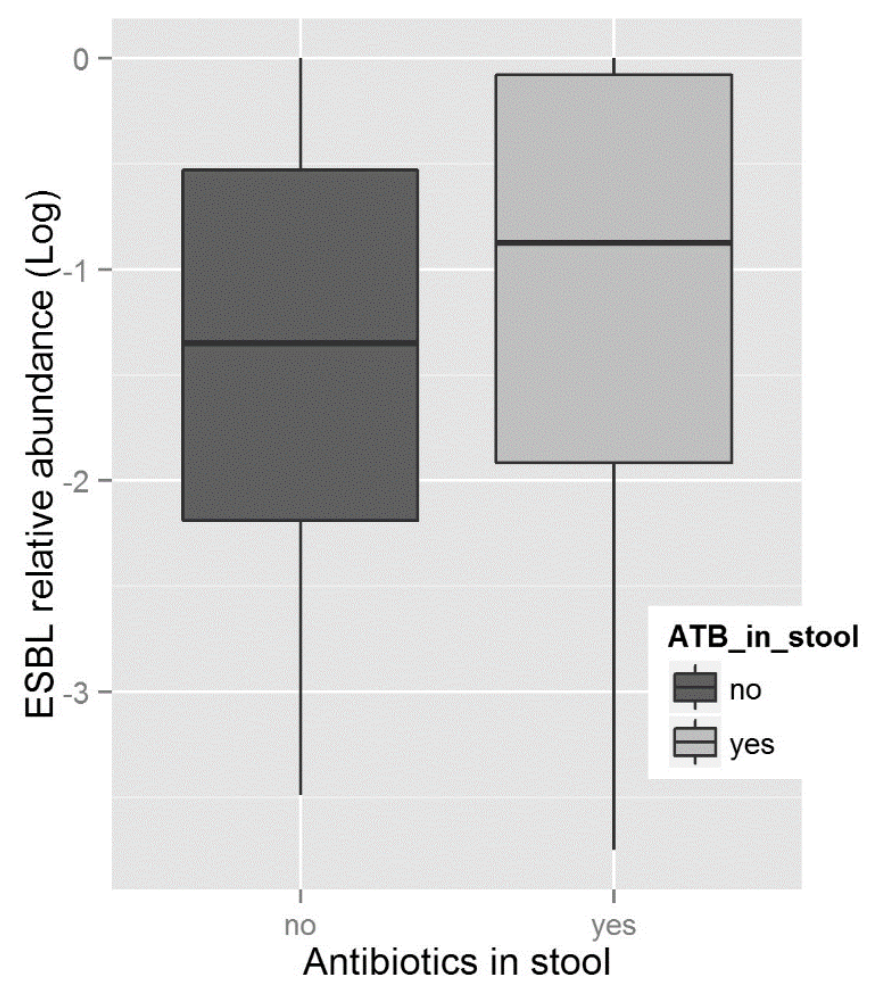

was the only independent risk factor associated with ESBL-E. coli carriage $(\mathrm{OR}=5.4$; 95\%CI [2.0-14.7]).

The mean ESBL-RA of 49 out of 57 patients was found to be higher in patients with antibiotic exposure than in patients without $(-1.1 \log [7,9 \%]$ vs. $-1.5 \log$ $[3.3 \%])$ but the difference was not significant $(\mathrm{p}=0.3)$ (Figure 1). Of note, ESBL-RA in patients without antibiotic exposure was similar to that observed in European women [1.1\%] [8]. ESBL-RA was not found to be associated with age, pregnancy, antibiotics use

Table 1. Risk factor analysis for fecal ESBL-producing E. coli carriage.

\begin{tabular}{|c|c|c|c|c|c|}
\hline & $\begin{array}{l}\text { ESBL - E. coli } \\
\text { carriers }\end{array}$ & $\begin{array}{l}\text { ESBL - } E . \text { coli } \\
\text { non carriers }\end{array}$ & $\boldsymbol{P}$ & $\begin{array}{l}\text { Multivariate } \\
\text { OR }\end{array}$ & $\boldsymbol{P}$ \\
\hline TOTAL & 57 & 29 & & & \\
\hline \multicolumn{6}{|l|}{ Season of the year } \\
\hline Dry & $39(8.4)$ & $23(79.3)$ & 0.3 & & \\
\hline Rainy & $18(31.6)$ & $6(20.7)$ & & & \\
\hline \multicolumn{6}{|l|}{ Patient's home origin } \\
\hline Yaoundé & $44(77.2)$ & $20(69)$ & & & \\
\hline Other regions & $13(22.8)$ & $9(31)$ & 0.4 & & \\
\hline Age (median) & $32[1-95]$ & 36 [10-89] & 0.7 & & \\
\hline Pregnancy & $15(26.3)$ & $11(37.9)$ & 0.3 & & \\
\hline Diabetes & $9(15.8)$ & $5(17.2)$ & $>0.99$ & & \\
\hline Hospitalization in the past three months & $4(7)$ & $6(20.7)$ & 0.08 & - & - \\
\hline Use of antibiotics in the past three months & $15(30.6)$ & $8(29.6)$ & $>0.99$ & & \\
\hline $\begin{array}{l}\text { Presence of antimicrobial activity in the stool } \\
\text { sample }\end{array}$ & $36(63.2)$ & $7(24.1)$ & 0.001 & $5.4(2.0-14.7)$ & $<0.001$ \\
\hline
\end{tabular}


during the last 3 months, hospitalization the last 3 months, diabetes or season.

\section{Discussion}

The rapid spread of CTX-M-type ESBL-E. coli in the community is not fully explained yet [9]. Such strains have emerged worldwide and have been reported in many countries. However, in African countries, data on ESBL-E. coli carriage in community patients are scarce. In our study, the prevalence of ESBL-E. coli carriage in stool was as high as $66.3 \%$. This is much higher than in a 2012 study in northern Cameroon which reported a prevalence of $23.1 \%$ among community outpatients [3] or in other African studies which reported rates of $30.9 \%$ in Niger [10], $10 \%$ in Senegal [11] and $7.3 \%$ in Tunisia [12]. This is even higher to what has been reported in China where a rate of $50.5 \%$ of CTX-M-E. coli [13] was described. In industrialized countries, carriage rates are much lower, as for example in France (6 \%) [14] or UK (11.3\%) [15]. The reasons why rates of carriage are generally higher in developing countries than in industrialized countries are still a matter of debate [2]. Many factors may have contributed to the high rates of resistance found in our patients. Mainly, our subjects were women with suspected UTI who were very often treated with antibiotics at the time of sampling, as demonstrated by the association between ESBL-E. coli carriage and the fecal antibiotic activity. That antibiotic treatment sharply increases ESBL fecal carriage rates has already been observed [16]. In the above-mentioned study from another part of Cameroon [3] where the rates of carriage were lower, subjects were not suspected of UTI and thus putatively less exposed to antibiotics at the time of sampling. This may explain why the rates, although already very high when compared to that in industrialized counties, were much lower than in our patients.

All of the ESBL strains isolated in this work were positive for CTX-M group 1, which is in accordance with the local epidemiology characterized by the predominance of the CTX-M-15 allele (belonging to group 1 CTX-M) [2]. Strains were also very often coresistant to other families of antibiotics such as aminoglycosides, fluoroquinolones and cotrimozaxole, in consistence with what was found elsewhere in Cameroon [3]. This situation may be the consequence of the high exposure of women with UTI to antibiotics and may favour dissemination and infections due to ESBL-producing Enterobacteriaceae.

One limitation of this study is that complementary data from the culture of the urine samples might have helped to assess the consequences of the ESBL carriage in our population. This issue, which has already been studied in other settings [6], was however not explored in this work.

\section{Conclusions}

In conclusion, we observed that the prevalence of carriage of ESBL-E. coli in women suspect of UTI is dramatically high in Yaoundé, Cameroon, and that this high prevalence was associated to recent antibiotic intake. All isolates produced a CTX-M-type ESBL. While all strains were susceptible to fosfomycin (a firstline antibiotic in UTI), most were resistant to the nonbeta-lactam antibiotics that are used to treat UTI infections, which may have very significant clinical consequences in case of pyelonephritis.

\section{Acknowledgements}

The authors wish to thank Dr. Aghokeng Avelin, Dr. Fosso, Mr Tchattad, Dr. Nkoa, Dr. Kouinche Adelaide, Dr. Valentine Ngum Ze, Mr. Pouma, Mr. Djamardin Mrs. Colette Ngono, Mr. Joseph Fokam and $\mathrm{Mr}$ Jean-Bapiste Ketchiewouo for their contribution to patient sampling process and for the preservation of strains.

\section{Financial support}

This work was supported in part by the Agence Universitaire de la Francophonie and the Bacteriological Laboratory of the University Training Hospital Bichat-Claude Bernard Paris.

\section{References}

1. Marcade G, Deschamps C, Boyd A, Gautier V, Picard B, Branger C, Denamur E, Arlet G (2009) Replicon typing of plasmids in Escherichia coli producing extended-spectrum beta-lactamases. J Antimicrob Chemother 63: 67-71.

2. Woerther PL, Burdet C, Chachaty E, Andremont A (2013) Trends in human fecal carriage of extended-spectrum betalactamases in the community: toward the globalization of CTX-M. Clin Microbiol Rev 26: 744-758.

3. Lonchel CM, Meex C, Gangoue-Pieboji J, Boreux R, Assoumou MC, Melin P, De Mol P (2012) Proportion of extended-spectrum b-lactamase-producing Enterobacteriaceae in community setting in Ngaoundere, Cameroon. BMC Infect. Dis. 12: 53.

4. Société Française de Microbiologie, Recommendations (2012). Available: http//www.sfm.microbiologie.org. Accessed 30 May 2012.

5. Ruimy R, Dos-Santo M, Raskine L, Bert F, MassonR, Elbaz S, Bonnal C, Lucet JC, Lefort A, Fantin B, Wolff M, Hornstein $\mathrm{M}$ and Andremont A (2008) Accuracy and potential usefulness of triplex real-time PCR for improving antibiotic treatment of patients with blood cultures showing clustered Gram-positive cocci on direct smears. J Clin Microbiol. 46: 2045-2051.

6. Woerther PL, Angebault C, Lescat M, Ruppe E, Skurnik D, Mniai AE, Clermont $\mathrm{O}$, Jacquier $\mathrm{H}$, Costa $\mathrm{AD}$, Renard $\mathrm{M}$, Bettinger RM, Epelboin L, Dupont C, Guillemot D, Rousset F, 
Arlet G, Denamur E, Djossou F, Andremont A (2010) Emergence and dissemination of extended-spectrum betalactamase-producing Escherichia coli in the community: lessons from the study of a remote and controlled population. J. Infect. Dis. 202: 515-523.

7. Messer JW, Leslie JE, Houghtby GA, Peeler JT, Barnett JE (1982) Bacillus stearothermophilus disc assay for detection of inhibitors in milk: collaborative study. J Assoc Off Anal Chem 65: 1208-1214.

8. Ruppe E, Lixandru B, Cojocaru R, Buke C, Paramythiotou E, Angebault C, Visseaux C, Djuikoue I, Erdem E, Burduniuc O, El Mniai A, Marcel C, Perrier M, Kesteman T, Clermont O, Denamur E, Armand-Lefevre L, Andremont A (2013) Relative fecal abundance of extended-spectrum-beta-lactamaseproducing Escherichia coli strains and their occurrence in urinary tract infections in women. Antimicrob Agents Chemother 57: 4512-4517.

9. Pitout JD, Nordmann P, Laupland KB, Poirel L (2005) Emergence of Enterobacteriaceae producing extendedspectrum beta-lactamases (ESBLs) in the community. J. Antimicrob. Chemother 56: 52-59.

10. Woerther PL, Angebault C, Jacquier H, Hugede HC, Janssens AC, Sayadi S, El Mniai A, Armand-Lefevre L, Ruppe E, Barbier F, Raskine L, Page AL, de Rekeneire N, Andremont A (2011) Massive increase, spread, and exchange of extended spectrum beta-lactamase-encoding genes among intestinal Enterobacteriaceae in hospitalized children with severe acute malnutrition in Niger. Clin Infect Dis 53: 677-685.

11. Ruppe E, Woerther PL, Diop A, Sene AM, Da Costa A, Arlet G, Andremont A, Rouveix B (2009) Carriage of CTX-M-15producing Escherichia coli isolates among children living in a remote village in Senegal. Antimicrob Agents Chemother 53: 3135-3137.

12. Ben Sallem R, Ben Slama K, Estepa V, Jouini A, Gharsa H, Klibi N, Saenz Y, Ruiz-Larrea F, Boudabous A, Torres C (2012) Prevalence and characterisation of extended-spectrum beta-lactamase (ESBL)-producing Escherichia coli isolates in healthy volunteers in Tunisia. Eur J Clin Microbiol Infect Dis 31: 1511-1516.

13. Li B, Sun JY, Liu QZ, Han LZ, Huang XH, Ni YX (2011) High prevalence of CTX-M beta-lactamases in faecal Escherichia coli strains from healthy humans in Fuzhou, China. Scand J. Infect Dis 43: 170-174.

14. Nicolas-Chanoine $\mathrm{MH}$, Gruson C, Bialek-Davenet S, Bertrand X, Thomas-Jean F, Bert F, Moyat M, Meiller E, Marcon E, Danchin N, Noussair L, Moreau R, Leflon-Guibout V (2012) 10 -Fold increase (2006-11) in the rate of healthy subjects with extended-spectrum beta-lactamase-producing Escherichia coli faecal carriage in a Parisian check-up centre. J Antimicrob Chemother 68: 562-568.

15. Wickramasinghe NH, Xu L, Eustace A, Shabir S, Saluja T, Hawkey PM (2012) High community faecal carriage rates of CTX-M ESBL-producing Escherichia coli in a specific population group in Birmingham, UK. J Antimicrob Chemother 67: 1108-1113.

16. Ruppe E, Pitsch A, Tubach F, de Lastours V, Chau F, Pasquet B, Lucet JC, Andremont A, Fantin B (2012) Clinical predictive values of extended-spectrum beta-lactamase carriage in patients admitted to medical wards. Eur J Clin Microbiol Infect. Dis. 31: 319-325.

\section{Corresponding author}

Paul-Louis Woerther, MD, PhD

Laboratoire de Microbiologie

Institut Gustave Roussy

114, rue Edouard Vaillant

94805 Villejuif cedexVillejuif

France

Phone: +33142114062

Fax: +33142115313

Email: paul-louis.woerther@gustaveroussy.fr

Conflict of interests: No conflict of interests is declared. 